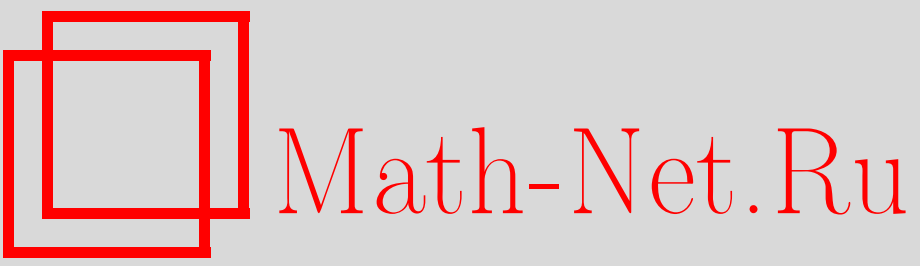

М. И. Купцов, В. А. Минаев, А. О. Фаддеев, С. Л. Яблочников, Об устойчивости интегрального многообразия системы обыкновенных дифференциальных уравнений в критическом случае, Итоги науки и техн. Сер. Соврем. мат. и ее прил. Темат. обз., 2019, том 168, 61-70

DOI: https://doi.org/10.36535/0233-6723-2019-168-61-70

Использование Общероссийского математического портала Math-Net.Ru подразумевает, что вы прочитали и согласны с пользовательским соглашением

http://www.mathnet.ru/rus/agreement

Параметры загрузки:

IP: 54.197 .130 .99

26 апреля 2023 г., $12: 30: 45$ 


\title{
ОБ УСТОЙЧИВОСТИ ИНТЕГРАЛЬНОГО МНОГООБРАЗИЯ СИСТЕМЫ ОБЫКНОВЕННЫХ ДИФФЕРЕНЦИАЛЬНЫХ УРАВНЕНИЙ В КРИТИЧЕСКОМ СЛУЧАЕ
}

\author{
() 2019 г. М. И. КУПЦОВ, В. А. МИНАЕВ,
} А. О. ФАДДЕЕВ, С. Л. ЯБЛОЧНИКОВ

\begin{abstract}
АннотАция. Рассматривается задача устойчивости ненулевых интегральных многообразий нелинейной конечномерной системы обыкновенных дифференциальных уравнений, правая часть которой является периодической вектор-функцией по независимой переменной и содержит параметр. Предполагается, что у изучаемой системы имеется тривиальное интегральное многообразие при всех значениях параметра, а соответствующая линейная подсистема не обладает свойством экспоненциальной дихотомии. Целью работы является нахождение достаточных условий существования в окрестности состояния равновесия системы ненулевого интегрального многообразия, а также условий его устойчивости и неустойчивости. Для этой цели на основе идей метода функций Ляпунова и метода преобразующей матрицы строятся операторы, позволяющие свести решение указанной задачи к поиску их неподвижных точек.
\end{abstract}

Ключевъе слова: метод функций Ляпунова, метод преобразующей матрицы, устойчивость интегрального многообразия, система обыкновенных дифференциальных уравнений, операторное уравнение.

\section{ON THE STABILITY OF INTEGRAL MANIFOLDS OF A SYSTEM OF ORDINARY DIFFERENTIAL EQUATIONS IN THE CRITICAL CASE}

\author{
(c) 2019 M. I. KUPTSOV, V. A. MINAEV,
}

\author{
A. O. FADDEEV, S. L. YABLOCHNIKOV
}

\begin{abstract}
In this paper, we consider the stability problem for nonzero integral manifolds of a nonlinear, finite-dimensional system of ordinary differential equations whose right-hand side is a periodic vector-valued function with respect to an independent variable containing a parameter. We assume that the system possesses a trivial integral manifold for all values of the parameter and the corresponding linear subsystem does not possess the exponential dichotomy property. We find sufficient conditions for the existence of a nonzero integral manifold in a neighborhood of the equilibrium of the system and conditions for its stability or instability. For this purpose, based of the ideas of the Lyapunov method and the method of transform matrices, we construct operators that allow one to reduce the solution of this problem to the search for fixed points.
\end{abstract}

Keywords and phrases: Lyapunov method, method of transform matrices, stability of integral manifolds, system of ordinary differential equations, operator equation.

AMS Subject Classification: 34A34, 34C25, 34C45, 34D35 
1. Введение. Пусть система обыкновенных дифференциальных уравнений

$$
f(\nu, y, \dot{y}, t)=0
$$

для любых $\nu, t$ имеет состояние равновесия $y=0$ и в области $\Lambda$ ее решения существуют и единственны. Здесь и далее $f, y, \nu-(n+m)$-векторы, $\dot{y}=d y / d t, f(\nu, y, \dot{y}, t+T) \equiv f(\nu, y, \dot{y}, t)$, $\Lambda=\Lambda_{1} \times \Lambda_{2}, \Lambda_{1}=\left\{y:\|y\| \leqslant \Delta_{1}\right\} \subset \mathbb{R}^{n+m}, \Lambda_{2}=\left\{\nu:\|\nu\| \leqslant \Delta_{2}\right\} \subset \mathbb{R}^{n+m}, \Delta_{1}, \Delta_{2}$ - константы, $\mathbb{R}^{p}$ - стандартное евклидово пространство размерности $p$ и $\|\cdot\|$ - евклидова норма в $\mathbb{R}^{p}$.

Предположим, что замена переменных

$$
y=\Gamma(\varepsilon, x, \phi, t), \quad \nu=\xi(\varepsilon)
$$

приводит систему (1) к виду

$$
\dot{x}=X(\varepsilon, x, \phi, t) \cdot x, \quad \dot{\phi}=\Phi(\varepsilon, x, \phi, t),
$$

где $\Gamma, \xi, \varepsilon-(n+m)$-векторы, $X-(n \times n)$-матрица, $x-n$-вектор, $\phi, \Phi-m$-векторы. Кроме того, будем полагать, что система

$$
\dot{x}=X(0,0, \phi, t) \cdot x, \quad \dot{\phi}=\Phi(0,0, \phi, t)
$$

имеет $m$-параметрическое семейство ненулевых $k T$-периодических решений $x=\bar{x}\left(\phi_{0}, t\right), \phi=$ $\bar{\phi}\left(\phi_{0}, t\right)$.

Определение 1. Будем говорить, что у системы (1) существует $n$-мерное нетривиальное периодическое интегральное многообразие $\Psi\left(\phi_{0}, t\right)$, если для всех $\phi_{0}$ найдется такое значение $\nu_{0}=\xi\left(\varepsilon_{0}\right)$ параметра $\nu$, при котором

$$
f\left(\xi\left(\varepsilon_{0}\right), \Gamma\left(\varepsilon_{0}, \Psi\left(\phi_{0}, t\right), \phi^{\Psi}\left(\phi_{0}, t\right), t\right), \dot{\Gamma}\left(\varepsilon_{0}, \Psi\left(\phi_{0}, t\right), \phi^{\Psi}\left(\phi_{0}, t\right), t\right), t\right) \equiv 0,
$$

причем $\Psi\left(\phi_{0}, t\right)$ не обращается в нуль ни при каких значениях $\phi_{0}$ и $t$, является $\omega$-периодическим по компонентам $m$-вектора $\phi_{0}, k T$-периодическим по $t$, где $k$-натуральное число, $\omega=\operatorname{colon}\left(\omega_{1}, \omega_{2}, \ldots, \omega_{m}\right), \phi=\phi^{\Psi}\left(\phi_{0}, t\right)$ определяет интегральную кривую на многообразии.

Ставятся задачи изучения проблем существования и устойчивости нетривиального периодического интегрального многообразия системы (1) вблизи ее состояния равновесия.

Вопросы существования и устойчивости (стабильности) инвариантных множеств систем дифференциальных уравнений имеют существенное значение для многих прикладных исследований. Замечательно, что области таких исследований все более и более расширяются и охватывают не только технические и естественнонаучные проблемы, но и философию, социальные и гуманитарные направления. Такие понятия, как нелинейность, бифуркация, аттрактор стали в определенном смысле уже философскими категориями, с помощью которых описывается не только «внешний», но и внутренний мир индивидуума (см. [14]). При моделировании реальных процессов инвариантная поверхность, имеющая меньшую размерность, позволяет упростить рассматриваемые системы, а также изучить их типичные свойства и получить дополнительную информацию об их состоянии. Особенно это характерно для устойчивых многообразий, «притягивающих» к себе траектории с течением времени. Впрочем, и «нестабильные» состояния, представляющие собой сложную смесь устойчивых и неустойчивых траекторий, все чаще возникают в практических моделях. Поэтому изучение интегральных многообразий, поведения траекторий на них и вблизи них позволяет решать многие задачи в различных областях: от теории нелинейных колебаний до диссипативных структур (см., например, [6, с. 6-9]). Однако разнообразие конкретных систем дифференциальных уравнений, возникающих в прикладных исследованиях, порождает трудности в получении общих эффективных методов исследования интегральных многообразий (особенно в критических случаях), что и обуславливает актуальность данной работы.

$\mathrm{K}$ наиболее продуктивным методам изучения интегральных многообразий следует отнести метод малого параметра, точечных отображений, усреднения и метод интегральных многообразий. Метод интегральных многообразий, разработанный Н. Н. Боголюбовым, Ю. А. Митропольским и А. М. Самойленко (см. $[2,9,11])$, состоит в построении функции Грина и успешно применяется для многих систем вида (3) (см., например, $[8,15]$ ). Однако в данном случае этот подход 
реализовать не удается, поскольку система (3) при всех значениях параметра имеет нулевое интегральное многообразие, а система (4) - параметрическое семейство периодических решений. Указанные условия удается обойти лишь с помощью нахождения решения вспомогательного векторного (бифуркационного) уравнения и перехода в его окрестность (см. [1,3]).

Изложенные в данной статье результаты получены на основе модификации предложенного в $[6,12]$ метода преобразующей матрицы и метода функций Ляпунова, применение которых позволили получить новые достаточные условия существования как устойчивого, так и неустойчивого локального интегрального многообразия системы (1). Полученные результаты развивают и обобщают работы $[4-7,12,13]$.

2. Существование интегрального многообразия. Пусть $F(\phi, t) \in \Omega_{1}, \varepsilon(\phi) \in \Omega_{2}-\omega$-периодические по компонентам вектора $\phi$ ограниченные соответственно числами $\delta_{10}$ и $\delta_{20}$ векторфункции, удовлетворяющие условию Липшица:

$$
\begin{gathered}
\left\|F\left(\phi_{1}, t_{1}\right)-F\left(\phi_{2}, t_{2}\right)\right\| \leqslant \delta_{11}\left\|\phi_{1}-\phi_{2}\right\|+\delta_{12}\left|t_{1}-t_{2}\right|, \\
\left\|\varepsilon\left(\phi_{1}\right)-\varepsilon\left(\phi_{2}\right)\right\| \leqslant \delta_{21}\left\|\phi_{1}-\phi_{2}\right\|,
\end{gathered}
$$

имеющие соответственно размерность $n$ и $l, 0<l \leqslant n+m, F(\phi, t)-k T$-периодическая по $t$,

$$
\|F(\phi, t)\|=\left[\sum_{i=1}^{n} \sup _{\substack{\phi_{j} \in\left[0, \omega_{j}\right] \\ t \in[0, k T]}}\left|F_{i}(\phi, t)\right|\right]^{1 / 2}, \quad\|\varepsilon(\phi)\|=\left[\sum_{i=1}^{l} \sup _{\phi_{j} \in\left[0, \omega_{j}\right]}\left|\varepsilon_{i}(\phi)\right|\right]^{1 / 2} .
$$

Отметим, что если для множеств $\Omega_{i}$ ввести указанную норму, то они становятся выпуклыми компактами (см. [6, с. 15]).

Для решения дифференциального уравнения

$$
\dot{\phi}=\Phi\left(\varepsilon\left(\phi_{0}\right), F\left(\phi_{0}, t\right), \phi, t\right),
$$

удовлетворяющего начальным данным $\phi(0)=\phi_{0}$, примем обозначение $\phi_{t}^{F}$. Пусть, кроме того, $Y_{\varepsilon}^{F}\left(\phi_{0}, t\right)$ - матрицант уравнения

$$
\dot{x}=X\left(\varepsilon\left(\phi_{0}\right), F\left(\phi_{0}, t\right), \phi_{t}^{F}, t\right) \cdot x .
$$

Здесь и далее $F\left(\phi_{0}, t\right) \in \Omega_{1}, n+m-l$ значений компонентов вектора $\varepsilon$ приняты равными 0 , а вместо оставшихся $l$ значений в уравнения системы (3) подставлены элементы функции $\varepsilon\left(\phi_{0}\right) \in$ $\Omega_{2}$.

Определение 2. Неособенную функциональную $(n \times n)$-матрицу $Q_{\varepsilon}^{F}\left(\phi_{0}\right)$ с постоянным определителем, непрерывную по всем своим переменным и $\omega$-периодическую по компонентам вектора $\phi_{0}$, будем называть преобразующей матрицей системы (3), если у матрицы

$$
\left(Y_{\varepsilon}^{F}\left(\phi_{0}, k T\right)-I_{n}\right) \cdot Q_{\varepsilon}^{F}\left(\phi_{0}\right)
$$

имеется столбец $q_{\varepsilon}^{F}\left(\phi_{0}\right)$, тождественно не обращающийся в нуль. Здесь $I_{n}-$ единичная $(n \times n)$ матрица.

Введем обозначения

$$
\mathrm{X}=\left\{x:\|x\| \leqslant \delta_{1}\right\} \subset \mathbb{R}^{n}, \quad \mathrm{E}=\left\{\varepsilon:\|\varepsilon\| \leqslant \delta_{2}\right\} \subset \mathbb{R}^{n+m}, \quad \Theta=\left\{\phi: \phi_{j} \in\left[0, \omega_{j}\right]\right\} .
$$

Пусть $\Gamma(\varepsilon, x, \phi, t) \neq 0$ при $x \neq 0$,

$$
\begin{aligned}
& \Gamma(\varepsilon, 0, \phi, t) \equiv 0, \\
& \Gamma(\varepsilon, x, \phi, t) \rightarrow 0
\end{aligned}
$$

при $\|x\| \rightarrow 0$ равномерно относительно совокупности переменных $\varepsilon, \phi, t$ в области $\mathbb{R}^{m+1} \times \mathrm{X} \times \mathrm{E}$.

Здесь и далее мы предполагаем, что правые части системы (3) являются $\omega$-периодическими по компонентам вектора $\phi$ и $T$-периодическими по независимой переменной $t \in \mathbb{R}$, непрерывны и обеспечивают существование и единственность решений системы (3) в области $\mathbb{R}^{m+1} \times \mathrm{X} \times$ $\mathrm{E}$ при достаточно малых $\delta_{1}$ и $\delta_{2}$. Это, в частности, означает, что замена переменных $(2)$ не 
только сохраняет свойства существования и единственности решений системы (1), но и является $\omega$-периодической по $\phi$ и $T$-периодической по $t$.

Рассмотрим систему уравнений

$$
q_{\varepsilon}^{F}\left(\phi_{0}\right)=0, \quad \int_{0}^{k T} \Phi\left(\varepsilon\left(\phi_{0}\right), F\left(\phi_{0}, t\right), \phi_{t}^{F}, t\right) d t=0 .
$$

Теорема 1. Пусть преобразующую матричу системь (3) удается построить так, что существуют такое число $l(0<l \leqslant n+m)$ и такой столбеи, $q_{\varepsilon}^{F}\left(\phi_{0}\right)$, при которых для нахождения решения системы (12) достаточно найти решение некоторой, вообще говорл, отличной от (12) системы l уравнений

$$
S_{\varepsilon}^{F}\left(\phi_{0}\right)=0,
$$

имеющей для каждой функиии $F\left(\phi_{0}, t\right) \in \Omega_{1}$ единственное решение $\varepsilon^{F}\left(\phi_{0}\right)$ из множества $\Omega_{2}$. Кроме того, пусть при $t \in[0 ; k T]$ выполнены неравенства

$$
\begin{gathered}
\left\|Y_{\varepsilon}^{F}\left(\phi_{0}, t\right) \cdot Q_{\varepsilon}^{F}\left(\phi_{0}\right)\right\| \leqslant r_{0}, \\
\left\|Y_{\varepsilon}^{F}\left(\phi_{0}^{*}, t^{*}\right) \cdot Q_{\varepsilon}^{F}\left(\phi_{0}^{*}\right)-Y_{\varepsilon}^{F}\left(\phi_{0}, t\right) \cdot Q_{\varepsilon}^{F}\left(\phi_{0}\right)\right\| \leqslant r_{1}\left\|\phi_{0}^{*}-\phi_{0}\right\|+r_{2}\left|t^{*}-t\right| .
\end{gathered}
$$

Тогда для любого вектора $\phi_{0} \in \mathbb{R}^{m}$ можно указать такое значение параметра $\nu$, что система (1) будет иметь ненулевое интегральное многообразие $\Psi\left(\phi_{0}, t\right) \in \Omega_{1}$ в окрестности состояния равновесия $y=0$.

Доказательство теоремы 1 опубликовано в [7,13], и поэтому здесь мы его не приводим. Отметим лишь, что для интегрального многообразия $\Psi\left(\phi_{0}, t\right)$, существование которого и доказывается в теореме 1, справедливо равенство

$$
\Psi\left(\phi_{0}, t\right)=Y_{\varepsilon}^{\Psi}\left(\phi_{0}, t\right) \cdot Q_{\varepsilon}^{\Psi}\left(\phi_{0}\right) \cdot C,
$$

где все элементы постоянного $n$-вектора $C$ равны нулю, кроме элемента, соответствующего номеру столбца $q_{\varepsilon}^{\Psi}\left(\phi_{0}\right)$, который равен произвольной константе $c$. При этом значения параметра $\varepsilon$ удовлетворяют уравнению (13), а, следовательно, и $(12)$, в которых $F\left(\phi_{0}, t\right) \equiv \Psi\left(\phi_{0}, t\right)$. Тогда выполнены соотношения

$$
\lim _{c \rightarrow 0} \frac{\left\|\Psi\left(\phi_{0}, t\right)\right\|}{c} \neq \infty, \quad \lim _{c \rightarrow 0}\left\|\varepsilon^{\Psi}\left(\phi_{0}\right)\right\|=0 .
$$

Технику построения преобразующей матрицы продемонстрируем в теореме 2 , устанавливающей существование интегрального многообразия по свойствам правых частей системы (3). Случай, когда проблема существования локального интегрального многообразия системы (1) решается по линейным относительно компонент вектора $\varepsilon$ членам, подробно рассмотрен в $[4-6,12]$. Поэтому будем рассматривать случай, когда линейные члены по компонентам параметра $\varepsilon$ не позволяют решить проблему существования локального интегрального многообразия.

Теорема 2. Пусть в области $\mathbb{R}^{m+1} \times \mathrm{X} \times \mathrm{E}$ при достаточно мальх $\delta_{1}$ u $\delta_{2}$ функиии $X(\varepsilon, x, \phi, t), \Phi(\varepsilon, x, \phi, t)$ липшич-непрерывны по всем своим переменным, $X(\varepsilon, x, \phi, t)$ имеет квазитреугольный вид

$$
\begin{gathered}
X(\varepsilon, x, \phi, t)=\left(\begin{array}{cc}
X_{1}(\varepsilon, x, \phi, t) & X_{3}(\varepsilon, x, \phi, t) \\
\Xi & X_{2}(\varepsilon, x, \phi, t)
\end{array}\right), \\
X(\varepsilon, 0, \phi, t)=\operatorname{diag}\left(X_{1}(\varepsilon, 0, \phi, t), X_{2}(\varepsilon, 0, \phi, t)\right), \\
X_{1}(0,0, \phi, k T)=\mathrm{const}, \quad \operatorname{det}\left(\exp \left(X_{1}(0,0, \phi, k T)\right)-I_{n-p}\right) \neq 0, \\
X_{2}(\varepsilon, x, \phi, t)=\operatorname{diag}\left(\alpha_{1}(\varepsilon, x, \phi, t), \alpha_{2}(\varepsilon, x, \phi, t), \ldots, \alpha_{p}(\varepsilon, x, \phi, t)\right), \\
\Phi(\varepsilon, x, \phi, t)=\operatorname{colon}\left(\alpha_{p+1}(\varepsilon, x, \phi, t), \alpha_{p+2}(\varepsilon, x, \phi, t), \ldots, \alpha_{p+m}(\varepsilon, x, \phi, t)\right),
\end{gathered}
$$


причем для $\bar{p}$ номеров $і$ выполняются условия

$$
\begin{gathered}
\alpha_{i}(\varepsilon, x, \phi, t)=a_{i 1} \varepsilon_{1}+a_{i 2} \varepsilon_{2}+\cdots+a_{i n+m} \varepsilon_{n+m}+\bar{\alpha}_{i}(\varepsilon, x, \phi, t), \\
\lim _{\|\varepsilon\| \rightarrow 0} \frac{\left\|\bar{\alpha}_{i}(\varepsilon, 0, \phi, t)\right\|}{\|\varepsilon\|}=0,
\end{gathered}
$$

для остальных $p-\bar{p}+m$ номеров $i$ выполняются условия

$$
\begin{gathered}
\alpha_{i}(\varepsilon, x, \phi, t)=\left(\varepsilon_{j_{i}}\right)^{p_{i}}-\tilde{\alpha}_{i}(\varepsilon, x, \phi, t), \\
\lim _{\|\varepsilon\| \rightarrow 0} \frac{\left\|\tilde{\alpha}_{i}(\varepsilon, 0, \phi, t)\right\|^{p_{i}}}{\|\varepsilon\|}=0,
\end{gathered}
$$

$p_{i}$ - рачиональное число $\left(1<p_{i}\right), \tilde{\alpha}_{i}(\varepsilon, x, \phi, t)$ имеют непрерывные частные производные по всем своим переменным. Если при этом $(-1)^{p_{i}}=1$, то для соответствующих номеров $i$ функиии $\tilde{\alpha}_{i}(\varepsilon, x, \phi, t)$ являются в области $\mathbb{R}^{m+1} \times \mathrm{X} \times \mathrm{E}$ положительно определенными.

Пусть, кроме того, определитель $\bar{p} \times \bar{p}$-матрицы $\bar{A}$, составленной из $\bar{p}$ столбцов $(\bar{p} \times(n+m))$ матриць $A=\left(a_{i k}\right)$ с номерами, отличными от $j_{i}$, не равен 0 . Здесъ и далее $X_{1}(\varepsilon, x, \phi, t)$, $X_{2}(\varepsilon, x, \phi, t), X_{3}(\varepsilon, x, \phi, t)$ - соответственно $(n-p) \times(n-p)$-, $p \times p$-, $(n-p) \times p$-матрици, элементами постоянной $p \times(n-p)$-матрицъ $\Xi$ являются нули, $\bar{\alpha}_{i}(\varepsilon, x, \phi, t)$ и $\tilde{\alpha}_{i}(\varepsilon, x, \phi, t)-$ скалярные функиии, среди номеров $j_{i}$ нет одинаковых.

Тогда для любого вектора $\phi_{0} \in \mathbb{R}^{m}$ можно указать такое значение параметра $\nu$, что система (1) будет иметь ненулевое интегральное многообразие $\Psi\left(\phi_{0}, t\right) \in \Omega_{1}$ в окрестности состояния равновесия $y=0$.

Доказательство. В силу свойств правых частей системы $(3)$, решение $\phi_{t}^{F}$ уравнения (7) ограничено, удовлетворяет условию Липшица по всем своим переменным при $t \in[0 ; k T]$ и $\omega$-периодическое по начальным данным $\phi_{0}$. Тогда и матрицант $Y_{\varepsilon}^{F}\left(\phi_{0}, t\right)$ уравнения $(8)$ обладает точно такими же свойствами (см., например, [6, с. 29]).

Можно показать (см., например, [6, с. 34]), что для матрицанта уравнения (8) справедливо представление

$$
Y_{\varepsilon}^{F}\left(\phi_{0}, t\right)=\bar{Y}_{\varepsilon}^{F}\left(\phi_{0}, t\right)+\tilde{Y}_{\varepsilon}^{F}\left(\phi_{0}, t\right),
$$

в котором $\bar{Y}_{\varepsilon}^{F}\left(\phi_{0}, t\right)$ является матрицантом системы уравнений

$$
\dot{x}=X\left(\varepsilon\left(\phi_{0}\right), 0, \phi_{t}^{F}, t\right) \cdot x,
$$

а $\tilde{Y}_{\varepsilon}^{F}\left(\phi_{0}, t\right)$ ограничено, удовлетворяет условию Липшица по всем своим переменным при $t \in$ $[0 ; k T]$ с постоянными, которые можно сделать как угодно малыми за счет уменьшения числа $\delta_{10}$ (т.е. $\left.\left\|F\left(\phi_{0}, t\right)\right\| \leqslant \delta_{10}\right)$.

Поскольку матрица $X(\varepsilon, x, \phi, t)$ имеет квазитреугольный вид, то

$$
Y_{\varepsilon}^{F}\left(\phi_{0}, k T\right)-I_{n}=\left(\begin{array}{cc}
Y_{11}\left(\varepsilon, F, \phi_{0}\right) & Y_{12}\left(\varepsilon, F, \phi_{0}\right) \\
\Xi & Y_{22}\left(\varepsilon, F, \phi_{0}\right)
\end{array}\right),
$$

где матрицы $Y_{11}\left(\varepsilon, F, \phi_{0}\right), Y_{12}\left(\varepsilon, F, \phi_{0}\right), Y_{22}\left(\varepsilon, F, \phi_{0}\right)$ имеют соответственно типы $(n-p) \times(n-p)$, $(n-p) \times p, p \times p$. Тогда, в силу выполнения неравенства

$$
\operatorname{det}\left(\exp \left(X_{1}(0,0, \phi, k T)\right)-I_{n-p}\right) \neq 0
$$

уменьшением постоянных $\delta_{1}$ и $\delta_{2}$ можно достичь выполнения соотношения

$$
\left|\operatorname{det}\left(Y_{11}\left(\varepsilon, F, \phi_{0}\right)\right)\right| \geqslant a_{0} \neq 0 \text {. }
$$

Это означает, что существует обратная матрица $\left(Y_{11}\left(\varepsilon, F, \phi_{0}\right)\right)^{-1}$ к матрице $Y_{11}\left(\varepsilon, F, \phi_{0}\right)$, которая также ограничена, $\omega$-периодична по $\phi_{0}$ и удовлетворяет условию Липшица по $\phi_{0}$.

Пусть вектор $\bar{q}=\operatorname{colon}\left(\bar{q}_{1}, \bar{q}_{2}, \ldots, \bar{q}_{n-p}\right)$ определяется равенством

$$
\bar{q}=-\left(Y_{11}\left(\varepsilon, F, \phi_{0}\right)\right)^{-1} \times Y_{12}\left(\varepsilon, F, \phi_{0}\right) J_{1},
$$


где элементами постоянного $(p \times 1)$-вектора $J_{1}$ являются единицы. Тогда преобразующую матрицу системы (3) выберем в виде

$$
Q_{\varepsilon}^{F}\left(\phi_{0}\right)=\left(\begin{array}{cc}
I_{n-p} & \bar{Q} \\
\Xi & J_{p}
\end{array}\right)
$$

где среди элементов $((n-p) \times p)$-матрицы $\bar{Q}$ ненулевыми являются лишь элементы $\bar{q}_{1}, \bar{q}_{2}, \ldots, \bar{q}_{n-p}$ последнего столбца, а среди элементов $(p \times p)$-матрицы $J_{p}$ ненулевыми являются лишь элементы главной диагонали и последнего столбца, все равные 1.

Поскольку элементы вектора $\bar{q}$ представляют собой линейные комбинации элементов матриц $\left(Y_{11}\left(\varepsilon, F, \phi_{0}\right)\right)^{-1}$ и $Y_{12}\left(\varepsilon, F, \phi_{0}\right)$, то $Q_{\varepsilon}^{F}\left(\phi_{0}\right)$ ограничена, $\omega$-периодична по $\phi_{0}$ и удовлетворяет условию Липшица по $\phi_{0}$ и, значит, будет выполнено (14) и (15).

В силу выбора преобразующей матрицы $Q_{\varepsilon}^{F}\left(\phi_{0}\right)$ у последнего столбца $q_{\varepsilon}^{F}\left(\phi_{0}\right)$ матрицы $(9)$ будет ровно $p$ ненулевых компонент. Действительно, первые $n-p$ координат столбца $q_{\varepsilon}^{F}\left(\phi_{0}\right)$ примут вид $Y_{11}\left(\varepsilon, F, \phi_{0}\right) \bar{q}+Y_{12}\left(\varepsilon, F, \phi_{0}\right) J_{1} \equiv 0$, а остальные $p$ координат запишутся следующим образом: $Y_{22}\left(\varepsilon, F, \phi_{0}\right) J_{1}$. Диагональный вид $X_{2}(\varepsilon, 0, \phi, t)$ матрицы обеспечивает и диагональность матрицы $Y_{22}\left(\varepsilon, F, \phi_{0}\right)$.

Выберем теперь число $l=p+m$ и отметим, что из свойств функций $\alpha_{i}(\varepsilon, x, \phi, t)$ следует, что в системе (12) ровно $p-\bar{p}+m$ уравнений не содержат линейных по компонентам параметра $\varepsilon$ членов. Без ограничения общности рассуждений будем считать, что первые $\bar{p}$ уравнений системы (12) содержат члены, линейные по компонентам параметра $\varepsilon$, а остальные $p-\bar{p}+m$ уравнений - нет.

По условиям теоремы 2 каждое из этих $p-\bar{p}+m$ уравнений может быть представлено в виде $\varepsilon_{j_{i}}= \pm\left(f_{i}\left(\varepsilon, F, \phi_{0}\right)\right)^{1 / p_{i}}$, если $(-1)^{p_{i}}=1$, или в виде

$$
\varepsilon_{j_{i}}=\left(f_{i}\left(\varepsilon, F, \phi_{0}\right)\right)^{1 / p_{i}}
$$

если $(-1)^{p_{i}}=-1$. При этом функции $f_{i}\left(\varepsilon, 0, \phi_{0}\right)$ содержат члены более высокого порядка по $\varepsilon$, чем $p_{i}$.

Таким образом, оператор $S_{\varepsilon}^{F}\left(\phi_{0}\right)$ (см. (13)) зададим первыми $\bar{p}$ уравнениями системы $(12)$ и $l-\bar{p}$ уравнениями вида (17). Обозначим символом $\bar{\Xi}$ нулевую $((l-\bar{p}) \times \bar{p})$-матрицу, а символом $\overline{\bar{A}}-\bar{p} \times(l-\bar{p})$-матрицу, состоящую из $l-\bar{p}$ столбцов матрицы $A$, не вошедших в матрицу $\bar{A}$. Значит, система уравнений (13) может быть записана следующим образом:

$$
\tilde{A} \varepsilon\left(\phi_{0}\right)=Y\left(\varepsilon, F, \phi_{0}\right),
$$

где

$$
\tilde{A}=\left(\begin{array}{cc}
\bar{A} & \overline{\bar{A}} \\
\bar{\Xi} & I_{l-\bar{p}}
\end{array}\right)
$$

т.е. $\operatorname{det} \tilde{A} \neq 0$. Кроме того, функция $Y\left(\varepsilon, F, \phi_{0}\right)$ является $\omega$-периодической по $\phi_{0}$, ограниченной и удовлетворяет условию Липшица по $\phi_{0}$ с постоянными, которые можно сделать как угодно близкими к нулю за счет уменьшения чисел $\delta_{i k}$ (см. (5), (6)).

Тогда путем уменьшения чисел $\delta_{i k}$ легко убедиться, что оператор, задающий уравнение (13), является сжимающим и для каждой функции $F\left(\phi_{0}, t\right) \in \Omega_{1}$ переводит пространство $\Omega_{2}$ в $\Omega_{2}$. Этим фактом окончательно устанавливается выполнение всех условий теоремы 1 и, следовательно, существование локального ненулевого интегрального многообразия системы (1). Теорема 2 доказана.

3. Устойчивость и неустойчивость интегрального многообразия. Пусть теперь и далее выполнены условия теоремы 1 и, значит, у системы (1) при некоторых значениях параметра $\nu_{0}=\xi\left(\varepsilon_{0}\right)$ имеется интегральное многообразие $\Psi\left(\phi_{0}, t\right) \in \Omega_{1}, \Psi\left(\phi_{0}, 0\right)=\Psi_{0}$. Обозначим через $x\left(x_{0}, \phi_{0}, t\right), \phi\left(x_{0}, \phi_{0}, t\right)$ решение уравнения $(3)$, удовлетворяющее начальным данным $x\left(x_{0}, \phi_{0}, 0\right)=$ $x_{0}, \phi\left(x_{0}, \phi_{0}, 0\right)=\phi_{0}$. 
Теорема 3 (см. [13]). Если при $\varepsilon=\varepsilon_{0}$ существует знакоопределенная функиия $V(x, t)$, для которой функиия

$$
\frac{d V}{d t}=\frac{\partial V\left(x\left(x_{0}, \phi_{0}, t\right)-\Psi\left(\phi_{0}, t\right), t\right)}{\partial t}+\sum_{i=1}^{n} \frac{\partial V\left(x\left(x_{0}, \phi_{0}, t\right)-\Psi\left(\phi_{0}, t\right), t\right)}{\partial x_{i}}
$$

знакопостоянна и имеет знак, противоположный $к$ знаку $V(x, t)$, то при $\nu_{0}=\xi\left(\varepsilon_{0}\right)$ интегральное многообразие $\Psi\left(\phi_{0}, t\right)$ системы (1) устойчиво.

Заметим, что в отличие от [10] устойчивость здесь подразумевается относительно всех переменных $y$, причем задача устойчивости полностью решается по свойствам лишь части переменных $x$.

Справедливы и общие теоремы об асимптотической устойчивости и неустойчивости интегрального многообразия. Схемы доказательства этих фактов в целом повторяют классические рассуждения (см., например, [10]) с некоторыми модификациями, обусловленными спецификой рассматриваемой системы (1).

Теорема 4. Если при $\varepsilon=\varepsilon_{0}$ существует знакоопределенная функиия $V(x, t)$, для которой функиия (18) знакоопределена и имеет знак, противоположный к знаку $V(x, t)$, а сама функиия $V(x, t)$ допускает бесконечно малый высший предел, то при $\nu_{0}=\xi\left(\varepsilon_{0}\right)$ интегральное многообразие $\Psi\left(\phi_{0}, t\right)$ системы (1) устойчиво асимптотически.

Доказательство. Пусть $\varepsilon=\varepsilon_{0}$ и $V(x, t)$ положительна. Тогда из теоремы 3 следует устойчивость многообразия $\Psi\left(\phi_{0}, t\right)$. Значит, начальные данные $x_{0}$ можно выбрать настолько близкими к $\Psi_{0}$, что при $t \in[0 ;+\infty)$ выполнено неравенство $d V / d t<0$ (см. (18)) и функция

$$
V\left(x\left(x_{0}, \phi_{0}, t\right)-\Psi\left(\phi_{0}, t\right), t\right)
$$

убывает.

Если предположить, что разность (19) ограничена снизу, то

$$
\left\|x\left(x_{0}, \phi_{0}, t\right)-\Psi\left(\phi_{0}, t\right)\right\| \geqslant l_{1}>0
$$

и, следовательно, $d V / d t \leqslant-l_{2}, l_{2}>0$. Значит,

$$
V\left(x\left(x_{0}, \phi_{0}, t\right)-\Psi\left(\phi_{0}, t\right), t\right)=V\left(x_{0}-\Psi_{0}, 0\right)+\int_{0}^{t} \frac{d V}{d t} d t \leqslant V\left(x_{0}-\Psi_{0}, 0\right)-l_{2} t,
$$

что противоречит (20). Поэтому предположение об ограниченности снизу функции (19) неверно и

$$
\lim _{t \rightarrow+\infty} V\left(x\left(x_{0}, \phi_{0}, t\right)-\Psi\left(\phi_{0}, t\right), t\right)=0
$$

а тогда

$$
\lim _{t \rightarrow+\infty}\left\|x\left(x_{0}, \phi_{0}, t\right)-\Psi\left(\phi_{0}, t\right)\right\|=0 .
$$

Теперь справедливость теоремы 4 следует из тождества (10) и предела (11).

Определение 3. Функцию $y=\Gamma(\varepsilon, x, \phi, t)$, удовлетворяющую соотношениям (10) и (11), будем называть равномерно возрастающей no $x$, если для любого достаточно малого $\epsilon_{1}>0$ найдется такое $\epsilon_{2}>0$, что из неравенства $\|x\|>\epsilon_{2}$ вытекает неравенство $\|\Gamma(\varepsilon, x, \phi, t)\|>\epsilon_{1}$ сразу для всех $\varepsilon, \phi, t$ в области $\mathbb{R}^{m+1} \times \mathrm{X} \times \mathrm{E}$, где $\epsilon_{2}<\delta_{1}$.

Теорема 5. Если функиия $y=\Gamma(\varepsilon, x, \phi, t)$ равномерно возрастает по $x$ и при $\varepsilon=\varepsilon_{0}$ существует допускающая бесконечно малый высший предел функиия $V(x, t)$, для которой производнал (18) знакоопределена, а сама функция $V(x, t)$ в области $X \times \mathbb{R}$ при сколь угодно малой $\delta_{1}$ может принимать значения того же знака, что и производная (18), то при $\nu_{0}=\xi\left(\varepsilon_{0}\right)$ интегральное многообразие $\Psi\left(\phi_{0}, t\right)$ системы (1) неустойчиво. 
Доказательство. Пусть $\epsilon_{1}>0$ - произвольное сколь угодно малое число, $\varepsilon=\varepsilon_{0}$ и $d V / d t>0$. Значит, можно выбрать постоянную $\epsilon_{2}>0$ и начальные данные $x_{0}$ настолько близкими к $\Psi_{0}$, чтобы одновременно выполнялось неравенство

$$
\left\|\Gamma\left(\varepsilon_{0}, x_{0}, \phi_{0}, 0\right)-\Gamma\left(\varepsilon_{0}, \Psi_{0}, \phi_{0}, 0\right)\right\| \leqslant \epsilon_{1},
$$

где $\|\Gamma(\varepsilon, x, \phi, t)\|>\epsilon_{1}$ при $\|x\|>\epsilon_{2}, V\left(x_{0}-\Psi_{0}, 0\right)>0$. Поэтому

$$
\left\|x_{0}-\Psi_{0}\right\| \leqslant \epsilon_{2} \text {. }
$$

Предположим, что с течением времени неравенство (22) не нарушится. Тогда, поскольку $d V / d t>0$, имеем

$$
V\left(x\left(x_{0}, \phi_{0}, t\right)-\Psi\left(\phi_{0}, t\right), t\right)>V\left(x_{0}-\Psi_{0}, 0\right) \quad \text { при } t \in(0 ;+\infty)
$$

и, следовательно,

$$
\left\|x\left(x_{0}, \phi_{0}, t\right)-\Psi\left(\phi_{0}, t\right)\right\| \geqslant \epsilon_{3}>0 .
$$

Но тогда $d V / d t \geqslant \epsilon_{4}>0$ и, значит,

$$
V\left(x\left(x_{0}, \phi_{0}, t\right)-\Psi\left(\phi_{0}, t\right), t\right) \geqslant V\left(x_{0}-\Psi_{0}, 0\right)+\epsilon_{4} t,
$$

что противоречит ограниченности функции $V(x, t)$ в области $(22)$. Полученное противоречие доказывает, что с течением времени неравенство (22) нарушается и вместе с ним нарушается неравенство (21). Таким образом, интегральное многообразие $\Psi\left(\phi_{0}, t\right)$ системы $(1)$ неустойчиво. Теорема 5 доказана.

В качестве иллюстрации использования теорем 4 и 5 рассмотрим простейшие системы вида (1)(примеры к теореме 3 см. в [13]).

Пример 1. Положим

$$
\beta_{1}=\nu_{1}-\nu_{1}^{2}+y_{1}^{2}+y_{2}^{2}, \quad \beta_{2}=\sin t+\nu_{1}+\nu_{2}+y_{1}+y_{2}^{2} .
$$

Рассмотрим систему двух уравнений

$$
\dot{y}_{1}=\beta_{1} y_{1}-\beta_{2} y_{2}, \quad \dot{y}_{2}=\beta_{2} y_{1}+\beta_{1} y_{2},
$$

содержащую только скалярные величины. Замена $y_{1}=x \cos \phi, y_{2}=x \sin \phi, \varepsilon_{i}=\nu_{i}$ приводит уравнения (23) к виду (3):

$$
\dot{x}=\left(\varepsilon_{1}-\varepsilon_{1}^{2}+x^{2}\right) x, \quad \dot{\phi}=\sin t+\varepsilon_{1}+\varepsilon_{2}+x \cos \phi+x^{2} \sin ^{2} \phi,
$$

причем соответствующая линейная подсистема (4)

$$
\dot{x}=0, \quad \dot{\phi}=\sin t
$$

имеет однопараметрическое семейство ненулевых $2 \pi$-периодических решений

$$
x=1, \quad \phi=\phi_{0}+1-\cos t .
$$

Тогда уравнения (7) и (8) соответственно запишутся следующим образом:

$$
\begin{gathered}
\dot{\phi}=\sin t+\varepsilon_{1}\left(\phi_{0}\right)+\varepsilon_{2}\left(\phi_{0}\right)+F\left(\phi_{0}, t\right) \cos \phi+F^{2}\left(\phi_{0}, t\right) \sin ^{2} \phi, \\
\dot{x}=\left(\varepsilon_{1}\left(\phi_{0}\right)-\varepsilon_{1}^{2}\left(\phi_{0}\right)+F^{2}\left(\phi_{0}, t\right)\right) x,
\end{gathered}
$$

а матрицант уравнения (8)

$$
Y_{\varepsilon}^{F}\left(\phi_{0}, t\right)=\exp \left[\left(\varepsilon_{1}\left(\phi_{0}\right)-\varepsilon_{1}^{2}\left(\phi_{0}\right)\right) t+\int_{0}^{t} F^{2}\left(\phi_{0}, \tau\right) d \tau\right] .
$$

Выбирая теперь преобразующую матрицу $Q_{\varepsilon}^{F}\left(\phi_{0}\right) \equiv 1$, приходим к уравнению $q_{\varepsilon}^{F}\left(\phi_{0}\right)=0$ (см. (12)):

$$
\exp \left[2 \pi\left(\varepsilon_{1}\left(\phi_{0}\right)-\varepsilon_{1}^{2}\left(\phi_{0}\right)\right)+\int_{0}^{2 \pi} F^{2}\left(\phi_{0}, t\right) d t\right]-1=0,
$$


или

$$
\varepsilon_{1}\left(\phi_{0}\right)-\varepsilon_{1}^{2}\left(\phi_{0}\right)+\frac{1}{2 \pi} \int_{0}^{2 \pi} F^{2}\left(\phi_{0}, t\right) d t=0
$$

Введя обозначение

$$
D_{F}\left(\phi_{0}\right)=1+\frac{2}{\pi} \int_{0}^{2 \pi} F^{2}\left(\phi_{0}, t\right) d t
$$

получаем

$$
\begin{gathered}
\varepsilon_{1}^{F}\left(\phi_{0}\right)=\frac{1-\sqrt{D_{F}\left(\phi_{0}\right)}}{2}, \\
\varepsilon_{2}^{F}\left(\phi_{0}\right)=-\varepsilon_{1}^{F}\left(\phi_{0}\right)-\frac{1}{2 \pi} \int_{0}^{2 \pi}\left(F\left(\phi_{0}, t\right) \cos \phi_{t}+F^{2}\left(\phi_{0}, t\right) \sin ^{2} \phi_{t}\right) d t
\end{gathered}
$$

эти функции являются единственным решением уравнения (13) для каждой функции $F\left(\phi_{0}, t\right)$. Значит, по теореме 1 у системы (23) существует интегральное многообразие $\Psi\left(\phi_{0}, t\right)$, удовлетворяющее (16):

$$
\Psi\left(\phi_{0}, t\right)=c \exp \left[\left(\varepsilon_{1}^{\Psi}\left(\phi_{0}\right)-\left(\varepsilon_{1}^{\Psi}\left(\phi_{0}\right)\right)^{2}\right) t+\int_{0}^{t} \Psi^{2}\left(\phi_{0}, \tau\right) d \tau\right] .
$$

Можно показать, что в данном случае выполняется соотношение $\Psi\left(\phi_{0}, t\right) \equiv c$, т.е.

$$
\varepsilon_{1}^{\Psi}\left(\phi_{0}\right)-\left(\varepsilon_{1}^{\Psi}\left(\phi_{0}\right)\right)^{2} \equiv-c^{2}, \quad \varepsilon_{2}^{\Psi}\left(\phi_{0}\right)=-\varepsilon_{1}^{\Psi}\left(\phi_{0}\right)-\frac{c}{2 \pi} \int_{0}^{2 \pi}\left(\cos \phi_{t}^{\Psi}+c \sin ^{2} \phi_{t}^{\Psi}\right) d t .
$$

Выберем функцию $V(x, t) \equiv x^{2}$. Заметим, что в силу теоремы о существовании и единственности решений функция $x\left(x_{0}, \phi_{0}, t\right)$ того же знака, что и $\Psi\left(\phi_{0}, t\right) \equiv c$, и поэтому

$$
\frac{d V}{d t}=2(x-c)^{2}(x+c) x>0
$$

(см. (18)). Таким образом, из теоремы 5 следует, что интегральное многообразие $\Psi\left(\phi_{0}, t\right)$ неустойчиво.

Если же в системе (23) $\beta_{1}=\nu_{1}-\nu_{1}^{2}-y_{1}^{2}-y_{2}^{2}$, то

$$
\varepsilon_{1}^{\Psi}\left(\phi_{0}\right)-\left(\varepsilon_{1}^{\Psi}\left(\phi_{0}\right)\right)^{2} \equiv c^{2}
$$

и, значит,

$$
\frac{d V}{d t}=-2(x-c)^{2}(x+c) x<0,
$$

т.е. по теореме 4 многообразие $\Psi\left(\phi_{0}, t\right)$ асимптотически устойчиво.

Таким образом, удалось получить новые достаточные условия существования устойчивых и неустойчивых интегральных многообразий системы (1). При этом задача устойчивости (неустойчивости) по всем переменным $y$ решается по свойствам лишь части переменных $x$. Дальнейшее развитие изложенных в данной работе результатов видится в построении функций Ляпунова $V\left(x, \phi_{0}, t\right)$, зависящих от векторного параметра $\phi_{0}$, и доказательств для таких функций аналогов теорем 3,4 и 5 .

Авторы выражают искреннюю благодарность профессору М. Т. Терехину за благожелательное внимание к нашей работе. 


\section{СПИСОК ЛИТЕРАТУРЫ}

1. Бибиков Ю. Н. Многочастотные нелинейные колебания и их бифуркации. - Л.: Изд-во ЛГУ, 1991.

2. Боголюбов H. H. О некоторых статистических методах в математической физике. - Львов: Изд-во АН УCCP, 1945.

3. Волков Д. Ю. Бифуркация инвариантных торов из состояния равновесия при наличии нулевых характеристических чисел// Вестн. Ленингр. ун-та. - 1988. - 1, № 2. - С. 102-103.

4. Купцов М. И. Локальное интегральное многообразие систем дифференциальных уравнений, зависящих от параметра// Диффер. уравн. - 1999. - 35, № 11. - С. 1579-1580.

5. Купцов М. И. Существование интегрального многообразия системы дифференциальных уравнений// Диффер. уравн. - 1998. - 34, № 6. - С. 855.

6. Купцов М. И. Существование интегральных многообразий и периодических решений системы обыкновенных дифференциальных уравнений/ Дисс. на соиск. уч. степ. канд. физ.-мат. наук - Ижевск: Удмурт. гос. ун-т, 1997.

7. Купщов М. И., Терехин М. Т., Теняев В. В. К проблеме существования интегральных многообразий системы дифференциальных уравнений, не разрешенных относительно производных// Ж. Средневолж. мат. об-ва. - 2017. - 19, № 2. - С. 76-84.

8. Курбаншоев С. З., Нусайриев М. А. Построение оптимальных интегральных многообразий для нелинейных дифференциальных уравнений// Докл. АН Респ. Таджикистан. - 2014. - 57, № 11-12. C. $807-812$.

9. Митропольский Ю. А., Лыкова О. Б. Интегральные многообразия в нелинейной механике. - М.: Наука, 1973.

10. Румянцев В. В., Озиранер А. С. Устойчивость и стабилизация движения по отношению к части переменных. - М.: Наука, 1987.

11. Самойленко А. М. Элементы математической теории многочастотных колебаний. Инвариантные торы. - М.: Наука, 1987.

12. Kuptsov M. I. Local integral manifold of a system of differential equations// Differ. Equations. - 1998. 34, № 7. - P. 1005-1007.

13. Kuptsov M. I., Minaev V. A. Stability integral manifold of the differential equations system in critical case// J. Phys. Conf. Ser. - 2018. - 973. - 012055.

14. Prigogine I. The philosophy of instability// Futures. — 1989. — № 21. — P. 396-400.

15. Sobolev $V$. Slow integral manifolds and control problems in critical and twice critical cases// J. Phys. Conf. Ser. - 2016. - 727. - 012017.

Купцов Михаил Иванович

Рязанский государственный радиотехнический университет

E-mail: kuptsov_michail@mail.ru

Минаев Владимир Александрович

Московский государственный технический университет имени Н. Э. Баумана

E-mail: m1va@yandex.ru

Фаддеев Александр Олегович

Академия права и управления Федеральной службы исполнения наказаний России, Рязань

E-mail: fao1@mail.ru

Яблочников Сергей Леонтьевич

Академия права и управления Федеральной службы исполнения наказаний России, Рязань

E-mail: vvkfek@mail.ru 